\title{
BEAM STABILITY IN THE DRIVE-BEAM DECELERATOR OF CLIC USING STRUCTURES OF HIGH-ORDER SYMMETRY
}

\author{
A. Millich, A. Riche, D. Schulte, CERN, 1211 Geneva, Switzerland
}

\begin{abstract}
The RF power necessary to accelerate the main beam of the Compact Linear Collider (CLIC) is produced by decelerating a high-current drive beam in Power Extraction and Transfer Structures (PETS). The reference structure is not cylindrically symmetric but has longitudinal waveguides carved into the inner surface. This gives rise to a transverse component of the main longitudinal mode which can not be damped, in contrast to the transverse dipole wakefield. The field is non-linear and couples the motion of the particles in the two planes. Limits of the stability of the decelerated beam are investigated for different structures.
\end{abstract}

\section{INTRODUCTION}

The CLIC study investigates a possible future linear collider based on acceleration with a frequency of $30 \mathrm{GHz}$ [1]. The necessary power is produced by decelerating a highcurrent, low-energy beam which runs parallel to the main beam. This is done in 20 consecutive drive beam decelerators per main linac each of which is fed with a new beam [2]. During the deceleration the beam develops a large energy spread of a factor ten and is subject to transverse deflections due to wakefields. For reliable operation the beam stability in the decelerator is critical since the beam contains a large energy per pulse. In addition, the down-times of the 40 decelerators add to the total downtime.

\section{DECELERATOR MODEL}

The decelerators are on average $767 \mathrm{~m}$ long and consist of 550 PETS [3]. The layout of the drive beam decelerator is strongly coupled to that of the main beam accelerator. It consists of a simple FODO lattice with a constant quadrupole spacing of $1.115 \mathrm{~m}$. Between each pair of consecutive quadrupoles a power extraction structure is placed. It feeds two accelerating structures in the main linac. The gradients of the quadrupoles are varied along the beam line to achieve a constant phase advance of about $88^{\circ}$ per FODO cell for the lowest energy particles. The envelopes of the particles with higher energies will then automatically be smaller than those with lower energies [4].

\section{STRUCTURE MODEL}

The active length of the power extraction structures is $0.8 \mathrm{~m}$; longer structures would lower the main linac fill fac- tor. The inner bore of the structure is cylindrically symmetric except for the longitudinal waveguides that are cut into the surface. Here four structures are considered with four, six and eight waveguides, respectively, and a circularly symmetric one. Their main properties are listed in Table 1. The longitudinal and transverse wakefields can be well represented by a single mode each, with a frequency of $30 \mathrm{GHz}$ for the longitudinal and $24 \mathrm{MHz}$ less for the transverse mode. The group velocities of the wakefields are close to the speed of light, e.g. $\beta_{\perp} \approx \beta_{\|}=0.441$ for the four waveguide structure.

The power the structure extracts from the beam has to be $512 \mathrm{MW}$. This is simply given by the product of average deceleration of the beam particles times the current. The longitudinal wakefield $W_{\|}$is a function of the structure radius $a, W_{\|} \propto a^{-3}$ for the four-waveguide structure. So one can in principle choose a high-energy, low-current beam in a small aperture structure or vice versa. Simulations of the transverse wakefield have shown that the best beam stability in a given decelerator using four waveguide structures is achieved by choosing the highest beam current possible. The injector complex is expected to allow for bunch charges $q$ of up to $q=20 \mathrm{nC}$.

The high group velocity leads to a concentration of longitudinal and transverse wakefields at the end of the structure. In the simulation, the passage of a particle through the structure is therefore simulated in a number of steps. During each step the longitudinal and transverse field is assumed to be constant. But from step to step, the fields vary according to the field profiles in the structure. The transverse wakefields not only drain out of the structure but are damped with a quality factor $Q \approx 50$ [5].

Since the structure is not cylindrically symmetric, the longitudinal field varies with the transverse position.

$$
E_{\|}(r, \phi)=\sum_{i=0}^{\infty} 2 k_{i} \frac{r^{m_{s} i}}{a^{m_{s} i}} \cos (2 \pi s / \lambda) \cos \left(m_{s} i \phi\right) .
$$

For the number of waveguides $m_{s} \neq 0$ this corresponds to a transverse field which can be written as

$$
\begin{aligned}
E_{\perp}(r, \phi)= & \sum_{i=0}^{\infty} 2 k_{i} m_{s} i \frac{r^{m_{s} i-1}}{a^{m_{s} i}} \frac{\lambda}{2 \pi} \sin (2 \pi s / \lambda) \\
& {\left[-\vec{e}_{r} \cos \left(m_{s} i \phi\right)+\vec{e}_{\phi} \sin \left(m_{s} i \phi\right)\right] . }
\end{aligned}
$$

Here, $\phi=0$ lies in a symmetry plane in the centre of a waveguide. In contrast to the transverse wakefield, this effect also plays a role if the beam is perfectly centred in the structures. 


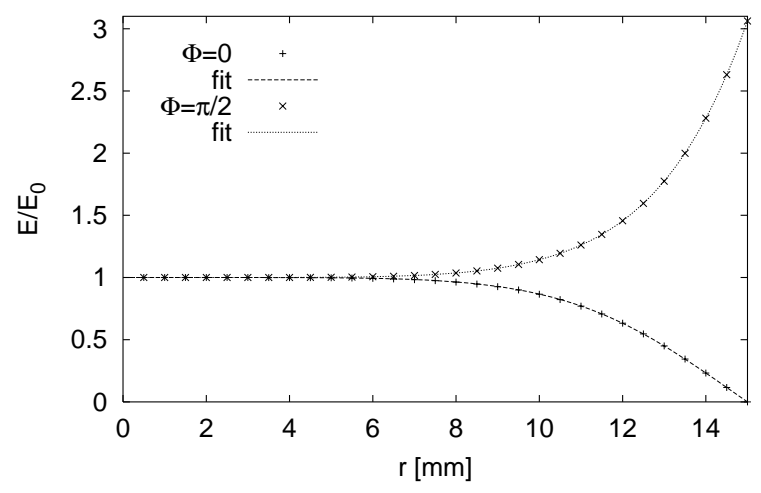

Figure 1: Fit of the field non-uniformity in the six waveguide structure.

Table 1: Parameters of the different power extraction structures used in the simulations. $R^{\prime} / Q$ is given in linac ohms. The last structure is circularly symmetric

\begin{tabular}{|c|c|c|c|c|c|}
\hline$m_{s}$ & & 4 & 6 & 8 & $\infty$ \\
$a$ & {$[\mathrm{~mm}]$} & 13 & 15 & 16 & 12 \\
$R^{\prime} / Q$ & {$[\Omega / \mathrm{m}]$} & 41 & 50.8 & 49.0 & 292.6 \\
$\hat{W}_{\perp}$ & {$\left[\mathrm{V} / \mathrm{pC} / \mathrm{m}^{2}\right]$} & 225 & 250 & 260 & 3170 \\
$\beta_{\|}$ & & 0.441 & 0.59 & 0.64 & 0.78 \\
$q_{b}$ & {$[\mathrm{nC}]$} & 16.1 & 16.7 & 17.7 & 8.0 \\
$E_{0}$ & {$[\mathrm{GeV}]$} & 1.34 & 1.29 & 1.22 & 2.76 \\
$\hat{G} / G$ & & 4.3 & 3.0 & 2.5 & 1.0 \\
$k_{1} / k_{0}$ & & 1.8 & 1.6 & 1.4 & - \\
$k_{2} / k_{0}$ & & 1.0 & 0.65 & 0.21 & - \\
\hline
\end{tabular}

For the six and eight waveguide structures the coefficients can be determined easily. Figure 1 shows the result of the fit in the two planes $\Phi=0$ and $\Phi=\pi / 2$ using eight coefficients. Only the first two are important, the others are quite small. In the case of the four waveguide structure, the fit is less reliable since the curves are not smooth due to the mesh used in the computations with MAFIA. Also in this case the first two coefficients are taken, even so the following ones are not much smaller. The most important effect is however expected from the lowest orders.

\section{BUNCH TRAIN}

The bunch train used to produce the RF power consists of four parts. In the main part, the flat top, the charge does not vary from bunch to bunch. It is used to produce the power during the passage of the main beam. It is preceded by a number of bunches in which the charge is increasing from bunch to bunch. This ramp is necessary to compensate the main linac beam loading by providing a field profile as if the first bunch of the main linac pulse had been preceded by others. An additional ramp of charge preceding the one described may be necessary if the drive beam injector cannot achieve the sudden change in charge. It is neglected in the following since its length and shape still have to be investigated. After the flat top, another ramp with decreas- ing charges will follow which is needed for beam loading compensation in the drive-beam accelerator, see [6].

The bunch length is assumed to be $\sigma_{z}=400 \mu \mathrm{m}$, the transverse emittances $\gamma \epsilon_{x}=\gamma \epsilon_{y}=150 \mu \mathrm{m}$. During the deceleration, particles can lose up to the $90 \%$ of their initial energy, while the first bunches will be hardly decelerated.

\section{ADDITIONAL ENERGY LOSS}

Due to the non-uniformity, off-axis particles can be decelerated more or less than on-axis. If the deceleration is too large these particles will be lost since they are over-focused by the quadrupoles. For a four-waveguide structure, the additional energy loss $\Delta E$ can be estimated as

$$
\Delta E=G \frac{3}{8} c_{0} \int_{0}^{L} \frac{\left\langle r^{4}(z)\right\rangle}{a^{4}} \mathrm{~d} z .
$$

Here, $L$ is the linac length, $\hat{r}(z)$ the maximum amplitude at position $z$ and the factor $3 / 8$ is due to the integration over the betatron motion. For the lowest energy particles the beta-function is constant, so the integration leads to

$$
\frac{\Delta E}{E_{0}-E_{f}}=\frac{3}{8} c_{0} \frac{\hat{r}^{5}-\check{r}^{5}}{5(\hat{r}-\check{r}) a^{4}} \frac{E_{0}}{E_{f}} .
$$

Here, $\hat{r}$ and $\check{r}$ are the minimal and maximal amplitudes in the first FODO cell. If $\Delta E / E_{f} \approx 0.3$ the lattice will be over-focusing. Assuming $k_{1} / k_{0}=3.3$ in the four waveguide structure (attributing the full amplitude of the nonuniformity to this value) a particle can be lost if it has an initial offset of about $8 \sigma$. Simulations confirm this value.

If consecutive structures are rotated by $\pi / m_{s}$ the lowest order term of the non-uniformity will about cancel. This will allow the particles to have even larger initial amplitudes without being lost.

\section{TRANSVERSE EFFECTS}

In order to stabilise the beam, it is useful to rotate every second structure by $\Phi=\pi / m_{s}$ [3]. Since the beta-function is significantly longer than the structures, the kick due to the lowest order in (1) averages out over a short distance. In Fig. 2, the maximum envelopes along the decelerator are shown for all three structures. A damping with $Q=50$ and $Q=200$ is assumed, the beam is offset by one sigma initially and has a size of $4 \sigma_{x}$ and $4 \sigma_{y}$. The envelope is normalised to the half-aperture. Even with $Q=200$ the beam is sufficiently stable in all structures. The ones with six and eight waveguides are better than the one with four due to the larger aperture and the smaller non-uniformity.

\section{AZIMUTHAL DEPENDENCE}

The kick a particle experiences due the field nonuniformity is purely radial in a symmetry plane, but elsewhere also has an azimuthal component. To investigate the different effects, a four sigma beam is used, containing test particles with Courant-Snyder Invariants $A_{x}=$ 

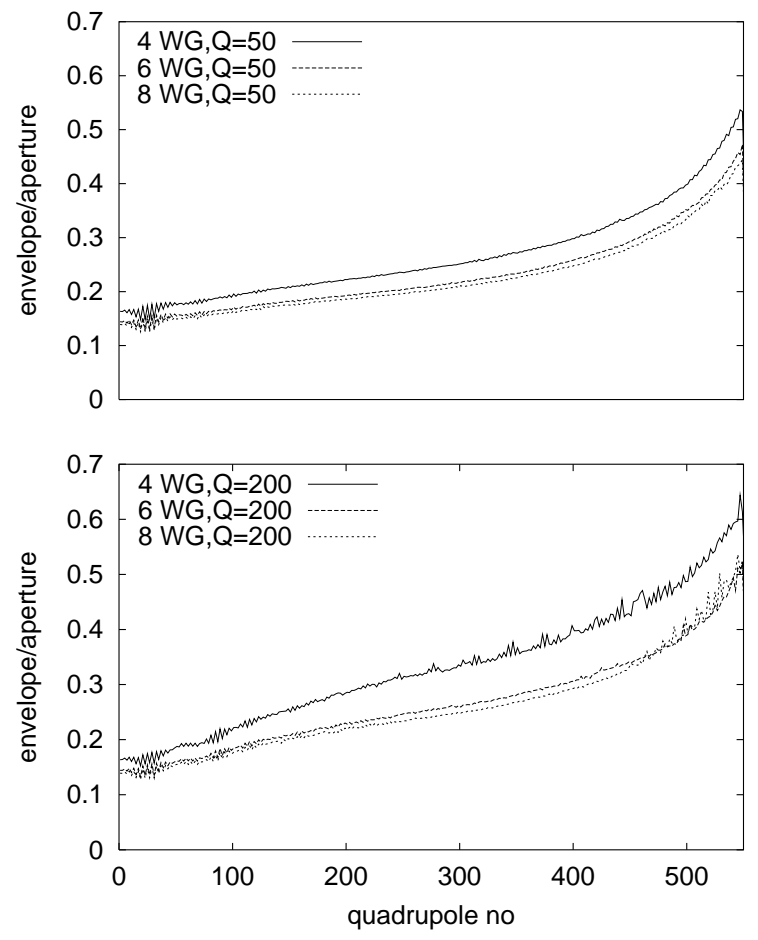

Figure 2: The normalised envelopes for the different structures with waveguides, the upper graph is for the expected damping $Q=50$, the lower for $Q=200$.

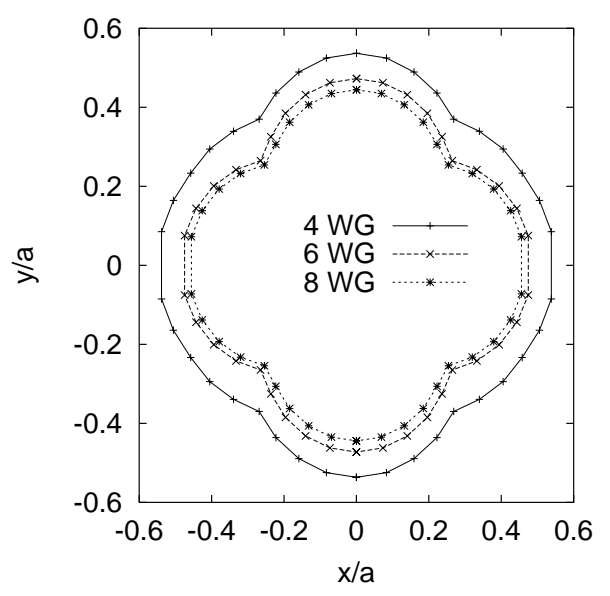

Figure 3: The envelopes in the different angles $\phi_{0}$.

$16 \cos ^{2}\left(\phi_{0}\right) \epsilon_{x}, A_{y}=16 \sin ^{2}\left(\phi_{0}\right) \epsilon_{y}$. The beam has an initial offset in the direction of this plane of one sigma: $\Delta_{x}=\cos \left(\phi_{0}\right) \sigma_{x}, \Delta_{y}=\cos \left(\phi_{0}\right) \sigma_{y}$. Figure 3 shows the maximal excursion of any particle for the different angles. The variation is essentially due to the optics only.

\section{CIRCULARLY SYMMETRIC STRUCTURE}

The circularly symmetric structure has no field inhomogeneities. Its transverse wakefield as well as its impedance

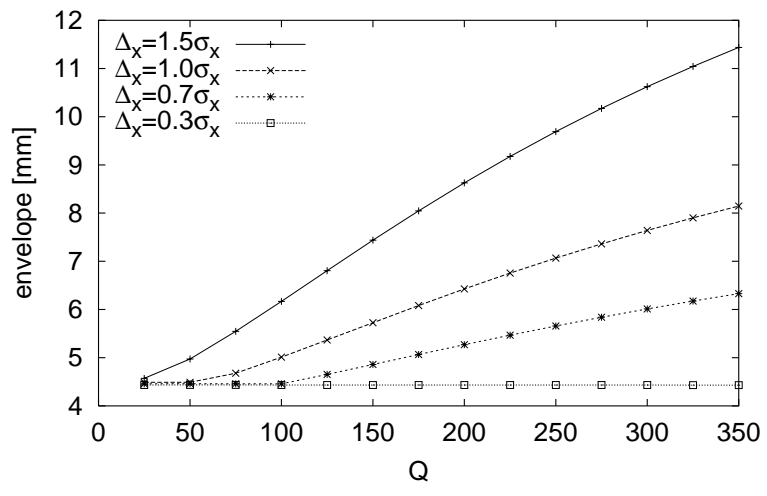

Figure 4: The beam envelopes for the circularly symmetric structure for damping with different $Q$.

are high. While it therefore seems natural to use a structure with larger diameter, it is very difficult to achieve a low group velocity in this case. Already at $r=12 \mathrm{~mm}$, it is very high: $\beta_{g}=0.78$. The initial energy of the beam is very high and its current significantly lower than in the former cases, see Table 1. In this case, it is more difficult to damp the transverse modes than in the former ones. First measurements indicate $Q \approx 150$ [7]. Figure 4 shows the maximal amplitudes of a four-sigma beam with different initial offsets for different values of $Q$. While the structure is clearly not excluded, the wakefield effect seems to be significantly stronger than in the previous ones.

\section{CONCLUSION}

Calculations of the beam stability in the drive beam decelerator have been performed for new transfer structures. The longitudinal and transverse effect of the non-uniformity of the longitudinal field have been included together with the usual wakefield effects. The stability improves compared to the previous case. Especially with the six and eight waveguide structures almost no effect of the nonuniformity is visible.

\section{REFERENCES}

[1] J.-P. Delahaye and 30 co-authors, "CLIC a 0.5 to $5 \mathrm{TeV}$ Compact Linear Collider.” EPAC 1998 and CERN-PS-98009-LP.

[2] H. Braun and 14 authors, "A new method of RF power generation for two-beam linear colliders." EPAC 1998 and CERN-PS-98-014-LP.

[3] A. Riche and D. Schulte. "The drive beam decelerator of CLIC". LINAC 98 and CERN-PS-98-043-LP

[4] A. Riche. "Maximum energy transfer efficiency in CLIC drive beam". CLIC-Note 266 (1994).

[5] L. Thorndahl. Private Communication.

[6] D. Schulte. "The drive beam accelerator of CLIC". LINAC 1998 and CERN-PS-98-042-LP.

[7] G. Carron and L. Thorndahl. Private communication. 